\section{Das Nationale Programm für nachhaltigen Konsum}

\author{
Ich bin 36 Jahre alt, verheiratet und habe ein Kind, bin studierte \\ Kulturwissenschaftlerin und praktizierende Beraterin \\ im Bereich Umwelt und Nachhaltigkeit. Vermutlich gehöre \\ ich zum sogenannten Milieu der LOHAS. Doch ist mein \\ Lebensstil nachhaltiger als andere? Von Romy Becker
}

la ch gehe im Bioladen meines Vertrauens einkaufen, ich achte dabei auf Siegel für die richtige Auswahl. Ich fahre oft mit dem Fahrrad - zumindest in meinem Kiez, besitze energieeffiziente Geräte, trenne meinen Müll, bemühe mich meine Lebensmittelreste möglichst gering $\mathrm{zu}$ halten, ich schätze Homeoffice und die neuen Möglichkeiten der Digitalisierung. Ich fahre gerne mit meiner Familie ins Berliner Umland und versuche, meinem Kind die Natur näher zu bringen.

Ich habe natürlich auch meine Schattenseiten: Ich liebe es, längere Strecken in der Stadt mit dem Taxi zu fahren, Geld für Kleider auszugeben, die ich nicht immer wirklich brauche, ich bestelle mir eine Pizza, bei der ich mir ziemlich sicher sein kann, dass der Belag nicht Bio ist, ich mag es gerne fern $\mathrm{zu}$ reisen und vergesse ab und $\mathrm{zu}$, das Licht auszuschalten. Doch im Rückblick der letzten zehn Jahre ist mein Verhalten in verschiedenen Lebensbereichen immer nachhaltiger geworden.

\section{Persönliche Perspektive als Ausgangspunkt}

Sensibilisiert wurde ich durch meinen Beruf und das hier erworbene Wissen zu den ökologischen und sozialen Auswirkungen meines Konsumverhaltens. Hier ist mir die Notwendigkeit eines nachhaltigen Lebensstils, auch mit Blick auf kommende Generationen deutlich vor Augen geführt worden. Doch der eigentliche Wandel in meinem Leben in den letzten Jahren kam vor allen durch die tägliche Praxis und das Ausprobieren zustande. Angefangen hat mein bewusster Konsum mit Lebensmitteln und Kosmetik. Wo wurden das Gemüse und das Obst angebaut? Wächst es um diese Zeit überhaupt in unseren Gefilden? Stammt das Fleisch aus der Massentierhaltung? Wie schmecken eigentlich die vegetarischen Aufstriche im Vergleich zu meiner geliebten Leberwurst? Mussten Tiere für mein Mascara leiden?

Durch die Auseinandersetzung mit Herkunft, Produktionsverfahren und Inhaltsstoffen entstanden immer mehr Fragen, die mich dazu bewogen haben, Alternativen auszuprobieren - auch für weitere Lebensbereiche, eigene Antworten zu finden und in meinem Leben immer mehr Alternativen zuzulassen und zu schätzen. Doch bin ich noch lange nicht am Ende angekommen. Familien mit geringerem Einkommen leben mitunter im Vergleich nachhaltiger - schaut man auf den Ressourcenverbrauch. Ein deutlicher Hinweis darauf, dass es nicht nur eine richtige Lebensweise gibt. Einen richtigen Lebensstil.

\section{Konsum mit Blick auf die Zukunft}

Aus diesem gewonnenen Erfahrungswissen leite ich wesentliche Chancen dafür ab, heute so zu konsumieren, dass die Bedürfnisbefriedigung heutiger und kommender Generationen unter Beachtung der natürlichen Grenzen unserer Erde nicht beeinträchtigt wird: Selbst in die Lage versetzt $z u$ werden, sich im Alltag Fragen zu stellen, die Freiheit zu besitzen, Verschiedenes auszuprobieren, durch die stetige Wiederholung von Alternativen andere Routinen auszubilden und das eigene Erfahrungswissen in weitere Lebensbereiche wirken $\mathrm{zu}$ lassen. Um diese tägliche Praxis zu ermöglichen, sind alle Akteure in der Gesellschaft gefragt, die direkt oder indirekt Einfluss auf den Alltag von uns allen haben: Bildungsinstitutionen, Unternehmen, Handel, Stiftungen, Nichtregierungsorganisationen, die Bürgerinnen und Bürger und selbstverständlich auch die Politik.

Die Bundesregierung hat im Februar 2016 das Nationale Programm für nachhaltigen Konsum verabschiedet, um Impulse zu setzen und alle relevanten Akteure $\mathrm{zu}$ aktivieren, unsere Gesellschaft nachhaltiger zu gestalten. Erstmalig und das kann an dieser Stelle nicht deutlich genug betont werden - wurden in einem ressortübergreifenden Prozess Leitlinien und Ziele definiert, übergreifende Handlungsansätze formuliert und über 170 konkrete Maßnahmen benannt, wie nachhaltiger Konsum in Deutschland gefördert werden kann.

\section{Der Plattformgedanke ist Programm}

Der Titel des Programms mag theoretisch und trocken anmuten, das Nationale Programm für nachhaltigen Konsum ist klar auf die Praxis orientiert. Übergeordnetes Ziel ist, einen substanziellen Beitrag zur Erreichung der Nachhaltigkeitsziele zu leisten, wie sie in der Nationalen Nachhaltigkeitsstrategie festgelegt wurden.

Das Programm leistet eine praxisorientierte Konkretisierung der Nachhaltigkeitsstrategie in den Konsumfeldern Mobilität, Ernährung, Wohnen, Arbeiten, Bekleidung und Tourismus, in denen Zielkorridore und Maßnahmen definiert sind. Die folgenden Leitideen liegen den Empfehlungen des Programms dabei zugrunde: Ermöglichung eines nachhaltigen Konsums für Bürgerinnen und Bürger $u$.a. durch Bildung und transparente Informationen, die Beförderung 
des nachhaltigen Konsums von der Nische in den Mainstream auf nationalen und internationalen Märkten, Gewährleistung der Teilhabe an nachhaltigem Konsum für alle Bevölkerungsgruppen, Anwendung der Lebenszyklus-Perspektive auf Produkte und Dienstleistungen sowie die Verschiebung des Produktfokus zur Systemsicht und den Perspektivwechsel vom Verbraucher zum Nutzer.

Das Nationale Programm für nachhaltigen Konsum versteht sich als eine flexible Plattform, welche sowohl zur Ausweitung bisheriger erfolgreicher Instrumente und Ansätze dienen soll als auch zur Initiierung neuer Projekte. Zudem sind im Programm im Rahmen der Gründung eines Nationalen Netzwerkes Nachhaltiger Konsum auch Aktivitäten und Projekte Dritter intendiert, die bei der Entwicklung und Umsetzung des Programms für nachhaltigen Konsum konkrete Beiträge leisten.

\section{Nationales Netzwerk Nachhaltiger Konsum}

Das Nationale Netzwerk Nachhaltiger Konsum verfolgt in seiner Zielstellung, nachhaltigen Konsum als gesellschaftsübergreifende Gestaltungsaufgabe dauerhaft im öffentlichen Bewusstsein zu verankern, einen fachlichen Austausch zwischen allen beteiligten Akteuren zu gewährleisten, Synergien für die Verwirklichung nachhaltiger Konsummuster, Lebensstile und Märkte zu schaffen und einen Bezug zu Politikprozessen auf globaler Ebene herzustellen. Mitmachen kann jeder [1]. Das Netzwerk wird durch das Kompetenzzentrum Nachhaltiger Konsum betreut.

Das Nationale Programm für nachhaltigen Konsum stellt nicht das Ende eines Prozesses dar, sondern setzt Leitplanken für den notwendigen Strukturwandel in Wirtschaft und Gesellschaft in Richtung Nachhaltigkeit in Deutschland. Den Weg bauen können wir alle nur gemeinsam - durch Taten nicht allein durch Forderungen. Jeder ist aufgerufen, an der Gestaltung des Nationalen Programms für nachhaltigen Konsum mitzuwirken.

\section{Wandel als Ziel}

Wir müssen uns trauen, Probleme offen anzusprechen, wie es von Johannes Doms (Mitglied der Geschäftsführung bei Hipp) auf der ersten Netzwerkkonferenz am 23. März 2017 gefordert wurde, doch auch gleichsam ins Handeln kommen und Entwicklungsperspektiven darlegen. Wir sollten uns mehr zutrauen, ausprobieren, auch das Scheitern zulassen und am Ende zu einer Alltagspraxis gelangen, die uns Fragen erlaubt und Antworten finden lässt. Jeder auf seine, den zukünftigen Generationen gerechte Weise.

Das Nationale Programm für nachhaltigen Konsum sollte von allen Akteuren aus Politik, Handel, Industrie, Bildung, Wissenschaft sowie Zivilgesellschaft genutzt werden, bisherige Aktivitäten in diesem Bereich zu bündeln, Synergien zu schaffen und gemeinsam unter einem Dach fortzuentwickeln.

\section{Anmerkung}

[1] Anmeldung für das Nationale Netzwerk Nachhaltiger Konsum unter www.k-n-k.de/ nationales-netzwerk-nachhaltiger-konsum. Neben der Webseite des Kompetenzzentrums stehen den Akteuren verschiedene Experten-Workshops, Netzwerktreffen und Netzwerkkonferenzen für den Austausch zur Verfügung. Die erste Netzwerkkonferenz fand am 23. März 2017 in Berlin statt.

\section{AUTOREN + KONTAKT}

Romy Becker ist als Senior Associate mit adelphi verbunden und war als Projektleiterin u. a. verantwortlich für die laufende Implementierung des Nationalen Programms für nachhaltigen Konsum. Darüber hinaus ist sie als selbstständige Beraterin tätig.

adelphi. Tel.: +49 30 8900068-27, E-Mail: becker@adelphi.de

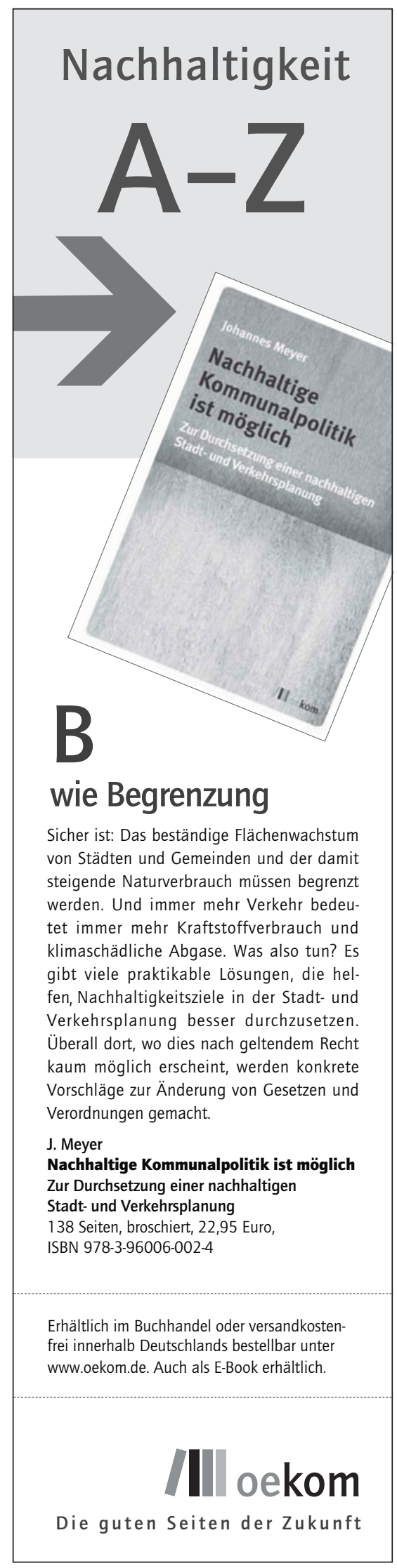

